\title{
An Edge Regeneration Method for Artifacts Removal in Compressed Image
}

\author{
Harpreet Kaur \\ Department of Computer \\ Science \& Engineering \\ DAVIET, Jalandhar
}

\author{
Parveen Kakkar \\ Department of Computer \\ Science \& Engineering \\ DAVIET, Jalandhar
}

\author{
Jagroop Singh, PhD \\ Department of Computer \\ Science \& Engineering \\ DAVIET, Jalandhar
}

\begin{abstract}
The region of image compression since it is applicable to various fields of image processing. The key goal of compression is to cut back the space for storage and decrease the transmission cost and maintain the visual quality of image. This paper presents fundamental approach for analysis and evaluating the image compression techniques. This paper describes the many techniques which can be used for image compression and describes about artefacts which can be consequence of compression and their reduction techniques. This paper presents a new Canny edge based restoration method to remove artefacts from compressed image. Firstly standard JPEG compression is used to compress the first image. Compressed image contains various visible artefacts like blurring artefacts, blocking artefacts and ringing artefacts. To attain better results than previous methods this paper proposed a new method. Experimental effects of proposed method by use of various performance parameters are good.
\end{abstract}

\section{Keywords}

JPEG Image compression, artefacts, algorithm to remove artefacts from compressed image

\section{INTRODUCTION}

Digital Images comes the thoughtful issue of storing and transferring the huge volume of data demonstrating the images because the uncompressed program data requires significant storage capacity and transmission bandwidth. Image Compression is a plotting from a complex dimensional space to a lesser dimensional space. Compressing [1] an image is significantly different than compressing raw binary data. Image compression [2] is also vital for reducing data transmission requirements of image and video content, which is particularly important given the ever increasing usage of image and video content over the Internet. The main goal of compression is to reduce the storage space and cut down the transmission cost and maintain visual quality. Compression [3] is the process of coding that will effectively reduce the total number of bits needed to represent certain information. Compression [3] is achieved by the removal of one or more of the three basic data redundancies:

\section{Coding Redundancy \\ 2. Inter-pixel Redundancy \\ 3. Psycho-visual Redundancy}

Coding redundancy is implemented by use of look up tables. Inter-pixel redundancy is based on those pixels values that have same or almost same values. Psycho-visual redundancy based on human vision.

The amount of data essential to represent an digital image makes broadcast slow and storage expensive. The amount of data used to represent these images needs to be reduced.
Basically image compression is done for efficient storage and broadcasting of digital image.

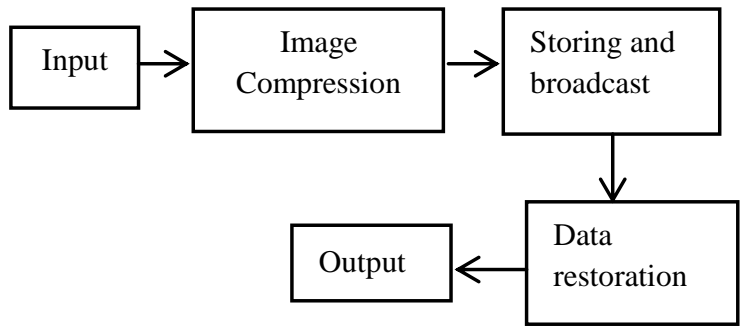

Fig.1 Image compression for storage and broadcast

\subsection{JPEG Compression}

JPEG compression is lossy image compression method of compression. The Joint Photographic Experts Group (JPEG) has been suggested it as a standard compression method for still images. JPEG uses an $8 \mathrm{X} 8$ pixel-block discrete cosine transform for stuffing information into limited transform coefficients. The block DCT method takes benefit of the local spatial correlation property of pictures and keep saves processing time. The individual processing of each image block makes visually blocking effects. JPEG was designed to compress color or gray-scale images.

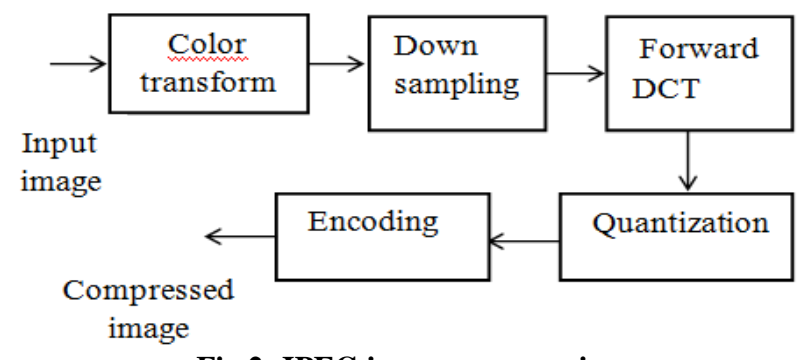

Fig.2. JPEG image compression

\subsection{Limitations of Compression}

When we compress the image by use of compression techniques it encounters artifacts in resultant image.

\section{Artifacts}

When image compressed by use of various techniques of image compression then compressed image may encounter visual distortion i.e. Compression artefacts. Artifacts are of various types, like, Blocking artefacts, Ringing artefacts, Blurring artefacts, color artefacts.

\section{Blocking Artifacts}

One of the most encountered disadvantages of the JPEG compression [3] is when we compress the image at very low bit rates then the compression can leave discontinuities of intensities between blocks known as blocking artifacts.JPEG can also lead to other visual artifacts such as degraded textures, 
blurring and distortion of edges i.e. with decreasing the bit rates will increase the occurrence of these visual artifacts [3].

\section{Blurring Artifacts}

Blurring in an image is cause of high spatial frequencies, which commonly occurs during filtering or compression. Blurring artifacts occurs all around the images.

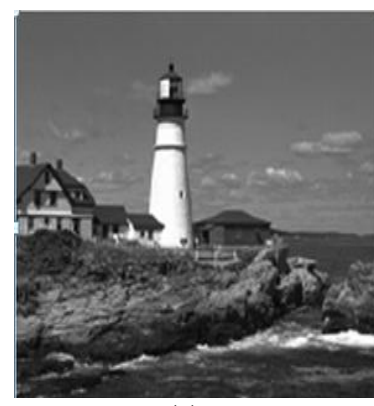

(a)

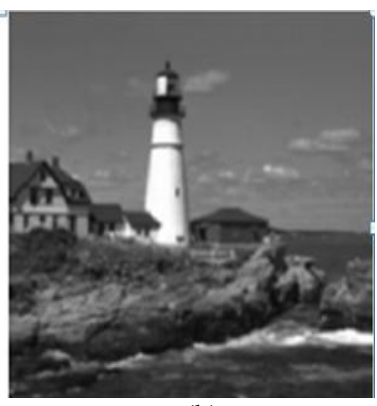

(b)
Fig.3 (a) Original image (b) Image with blurring artifacts

\section{Ringing artifacts}

Ringing artefacts is caused by heavy truncation on transform coefficients and can also come from improper image restoration operations. Mostly post-processing method is preferable to remove these artefacts.

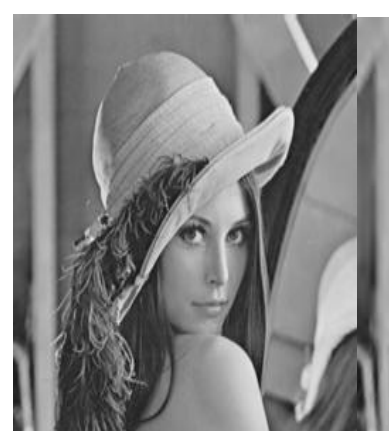

(a)

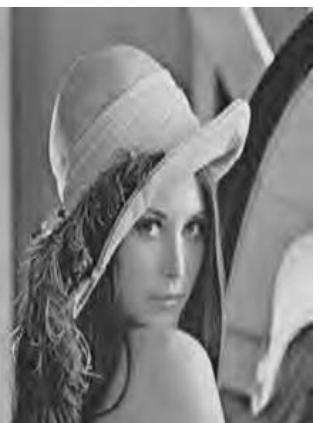

(b)
Fig.4 (a) Original image (b) Image with ringing artefacts

\section{Color artifacts}

The main difference between the color artifacts and non-color artifacts is the color of image.

Color artifacts are the colored distortion i.e. changed color of image or unwanted color of image.

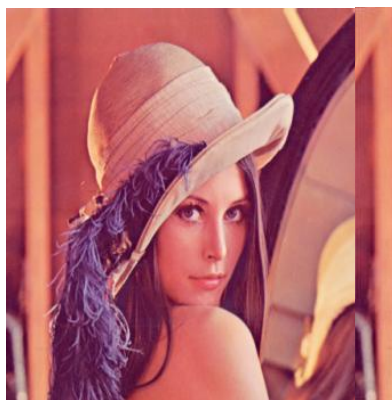

(a)

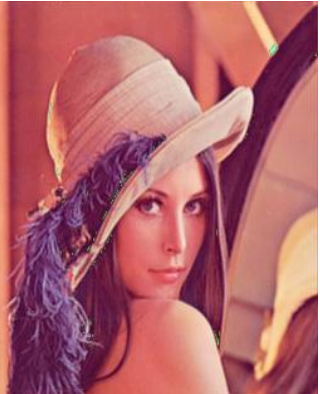

(b)
Fig.5 (a) Original image (b) Image with color artefacts

\section{PROPOSED WORK2.1 Edge preservation based restoration method}

Image compression has become very important tool in digital image processing. The main objective of the compression is to reduce the amount or unwanted data while retaining the information in the image. The goal behind is to save the amount of memory required to save the image(s) or to utilize network bandwidth in efficient manner. Transform-based compression [8] is extensively used for image compression. But transform based methods introduce blocking artifacts in the output image. The compression ringing artifacts [5] around edges can be efficiently removed using edge restoration as a post- processing. Most of the existing techniques are for gray scale images only. Thus, artifacts induced via quantization of the DCT components of the chrominance channels are not addressed. The use of the color artifacts removal techniques has also ignored in the existing research.

\section{ALGORITHM}

Artefact_removal (I)

Step1: Apply JPEG Compression to compress the image

$F(u, v)=\frac{1}{N} \sum_{x=0}^{N-1} \sum_{y=0}^{N-1} f(x, y)$

Give input image and then apply JPEG compression to compress the image. After compression i.e. output image contains artifacts so to remove these artifacts use edge regeneration.

Step2: Calculate image averaging to smooth block boundaries

For $\mathrm{i}=9: 8$ : row-1

For $\mathrm{j}=9: 1$ : Column-1

$I_{\text {avg }}(i, j)=(I(i-1, j)+13 * I(i, j)+I(i+1, j)) / 15$;

$I_{\text {avg }}(i-1, j)=(I(i-2, j)+13 * I(i-1, j)+I(i, j)) / 15$;

(3)

End

End

Smooth block boundaries the first step of de-blocking. We can smooth the block boundaries by using image averaging [22]. The $2^{\text {nd }}$ step is to use guided filter to reduce blocking artifacts. If we apply guided filter without smoothing the boundaries, the image may still have blocking artifacts around the boundaries [22].

Step3: Evaluate optimal regularization parameter of guided filter for smoothening

$\epsilon=0.0067 Q^{-0.7891}-0.0003$

The guided filter has two parameters: $\epsilon$ and $\alpha . \epsilon$ is regularization parameter and $\alpha$ specifies the local window radius. With increasing these parameters generally results in more smoothing. By decreasing these parameters results in images which might still contain visible blocking artifacts.

\section{Step4: Apply Guided image filter for removal of block boundaries}

Input: filtering input image I, radius $\mathrm{r}$, regularization $\epsilon$

Output: Filtering output q.

$$
\text { 1: } \operatorname{mean}_{I}=\frac{\sum(I)}{N}
$$




$$
\begin{gathered}
\operatorname{mean}_{p}=\frac{\sum(p)}{N} \\
\operatorname{corr}_{I}=\frac{\sum(I \cdot * I)}{N} \\
\operatorname{corr}_{I p}=\frac{\sum(I \cdot * p)}{N} \\
\text { 2: } \operatorname{var}_{1}=\operatorname{corr}_{1}-\frac{\sum(I)}{N} \cdot * \frac{\sum(I)}{N} \\
\operatorname{cov}_{I_{p}}=\operatorname{corr}_{I_{p}}-\frac{\sum(I)}{N} \cdot \frac{\sum(p)}{N} \\
\text { 3: } a=\operatorname{cov}_{I_{p}} \cdot /\left(\operatorname{var}_{I}+\epsilon\right) \\
b=\frac{\sum(p)}{N}-a \cdot * \frac{\sum(I)}{N}
\end{gathered}
$$

4: $\operatorname{mean}_{a}=\frac{\sum(a)}{N}$

$$
\operatorname{mean}_{b}=\frac{\sum(b)}{N}
$$$$
5: \mathrm{q}=\frac{\sum(a)}{N} * I+\frac{\sum(b)}{N}
$$

$\mathrm{q}=\operatorname{GIF}\left(I_{\text {avg }}\right)$

Guided image filter has good edge-preserving smoothing properties like bilateral filter but it does not suffer from the gradient reversal artifacts. The filtering output is locally a linear transform of the guidance image.

Guided image filter is edge preserving smoothing parameter. Example, The guide I identical to the filtering input $\mathrm{p}$. Consider the case where $I \equiv p$. In this case, $a_{k}=\sigma^{2}{ }_{k} /\left(\sigma^{2}{ }_{k}+\epsilon\right)$ and $b_{k}=\left(1-a_{k}\right) \mu_{k}$. It is clear that if $\epsilon=0$, then $a_{k}=1$ and $b_{k}=0$.

\section{Step5: Apply canny edge detection to identify strong edges}

In edge regeneration stage firstly we use canny edge detection to detect strong edges. In order to compute the strong edges, we use adaptive threshold [22] parameters for low and high threshold of the canny edge detection.

1. Compute $f_{x}$ and $f_{y}$

$$
\begin{gathered}
f_{x}=\frac{\partial}{\partial x}(f * G)=f * \frac{\partial}{\partial x} G=f * G_{x} \\
f_{x}=\frac{\partial}{\partial y}(f * G)=f * \frac{\partial}{\partial y} G=f * G_{y}
\end{gathered}
$$

$G(x, y)$ is the Gaussian function

$$
\begin{aligned}
& G_{x}(x, y) \text { is the derivate of } G(x, y) \text { w.r.t } x: G_{x}(x, y) \\
& \qquad=\frac{-x}{\sigma^{2}} G(x, y) \\
& \begin{aligned}
G_{y}(x, y) \text { is the derivate of } G(x, y) \text { w.r.t } y: G_{y}(x, y) \\
=\frac{-y}{\sigma^{2}} G(x, y)
\end{aligned}
\end{aligned}
$$

Canny has shown that the first derivative of the Gaussian closely approximates the operator that optimizes the product of signal-to-noise ratio and localization.

2. Compute the gradient magnitude

$$
\operatorname{magn}(i, j)=\sqrt{f_{x}^{2}+f_{y}^{2}}
$$

3. Apply non maximum suppression
For each pixal $(\mathrm{x}, \mathrm{y})$ do:

$$
\begin{gathered}
\text { if } \operatorname{magn}(i, j)<\operatorname{magn}\left(i_{1}, j_{1}\right) \operatorname{ormagn}(i, j)<\operatorname{magn}\left(i_{2}, j_{2}\right) \\
\text { then } I_{N}(i, j)=0 \\
\text { else } I_{N}(i, j)=\operatorname{magn}(i, j)
\end{gathered}
$$

To find the edge points, we need to find the local maxima of the gradient magnitude.

Broad ridges must be thinned so that only the magnitudes at the points of greatest local change remain. All values along the direction of the gradient that are not peak values of a ridge are suppressed. The algorithm performs edge linking as a byproduct of double threshold.

4. Apply hysteresis threshold

4.1. Produce two threshold images $I_{1}(i, j)$ and $I_{2}(i, j)$

4.2. Link the edges in $I_{2}(i, j)$

4.2.1. Look in $I_{1}(i, j)$ when a gap is found

4.2.2. By examining the 8 neighbours in $I_{1}(i, j)$, gather edge points from $I_{1}(i, j)$ until the gap has been bridged to an edge in $I_{2}(i, j)$.

$\mathrm{M}=$ canny $(\mathrm{q})$;

Step6: Evaluate adaptive threshold using OTSU method used in edge regeneration

In Otsu's method we exhaustively search for the threshold that minimizes the intra-class variance (the variance within the class), defined as a weighted sum of variances of the two classes:

$\sigma_{\omega}^{2}(t)=\omega_{1}(t) \sigma_{1}^{2}(t)+\omega_{2}(t) \sigma_{2}^{2}(t)$

Weights $\omega_{i}$ are the probabilities of the two classes separated by a threshold $t$ and $\sigma^{2}{ }_{i}$ are variances of these classes.

Step7: Evaluate $T_{r}$ using equation (5)

$[\mathrm{BW}, \mathrm{T}]=\operatorname{canny}\left(M,\left[1.5 * T_{r} 2.5 * T_{r}\right], 5\right)$;

if $\mathrm{Q}<11(6)$

$[\mathrm{BW}, \mathrm{T}]=\operatorname{canny}\left(M,\left[2 * T_{r} 2.75 * T_{r}\right], 5\right) ;$ if $\mathrm{Q} \geq 11 \& Q<$ 26

$[\mathrm{BW}, \mathrm{T}]=\operatorname{canny}(M, 0.99,0.00001)$;

Otherwise (8)

Step8: Apply edge regeneration to remove blurring artifacts

$Z(i, j) \mathrm{d}_{\mathrm{left}}=\left|Z(i, j)-\frac{Z(i, j-1)+Z(i, j-2)}{2}\right|$

$Z(i, j) \mathrm{d}_{\text {right }}=\left|Z(i, j)-\frac{Z(i, j+1)+Z(i, j+2)}{2}\right|$

$Z(i, j)=\frac{Z(i+1, j-1)+Z(i, j-1)+Z(i+1, j)+Z(i, j)}{4}$

$Z(i, j) \mathrm{d}_{\text {above }}=\left|Z(i, j)-\frac{Z(i-1, j)+Z(i-2, j)}{2}\right|$

$Z(i, j) \mathrm{d}_{\text {below }}=\left|Z(i, j)-\frac{Z(i+1, j)+Z(i+2, j)}{2}\right|$

In equation (9), we choose to compare the edge pixel against the average of the two pixel values to the left and right [22]. By computing the $d_{\text {left }}$ and $d_{\text {right }}$, we measure the similarity of two sides of the edge pixel to the pixel on the edge. After computing $d_{\text {left }}$ and $d_{\text {right }}$, if $d_{\text {left }}<d_{\text {right }}$, this signifies that 
the left side of the edge pixel is more similar to the edge pixel than the right side. We would then use the side which is similar to the edge pixel to remake the edge pixel and its neighbours [22]. Then we use the pixels on the side which is different from the edge to reduce the artifacts around the edge.

\section{Step9: Apply edge preservation based restoration} method

$$
\begin{gathered}
\text { if } B W(i, j)==1 \\
\mathrm{a} 1=(\mathrm{q} 1(\mathrm{i}, \mathrm{j}-1)+\mathrm{q} 1(\mathrm{i}, \mathrm{j}-2)) / 2 \\
\mathrm{~b} 1=(\mathrm{q} 1(\mathrm{i}, \mathrm{j}+1)+\mathrm{q} 1(\mathrm{i}, \mathrm{j}+2)) / 2 \\
\mathrm{X}=\mathrm{q} 1(\mathrm{i}, \mathrm{j}) ; \\
\mathrm{a}=\mathrm{X}-\mathrm{a} 1 ; \\
\mathrm{b}=\mathrm{X}-\mathrm{b} 1 ; \\
\mathrm{U}=\mathrm{q} 1(\mathrm{i}-4: \mathrm{i}+4, \mathrm{j}-4: \mathrm{j}+4) ; \\
\operatorname{hist}=\text { imhist }((\mathrm{U}), 10) ; \\
\operatorname{hist}=\operatorname{isfinite}(1 . / \mathrm{hist}) ; \\
\mathrm{b}=\left(\frac{\sum(\text { hist })}{\mathrm{N}}\right) * 10
\end{gathered}
$$

$$
\text { if } \mathrm{b} \leq \mathrm{t}
$$

Where $\mathrm{t}$ is threshold and it is 20

$$
\begin{aligned}
& q 3(i, j+1)=\left(\begin{array}{c}
q 2(i, j)+q 2(i-1, j+1)+ \\
q 2(i, j+1) \\
+q 2(i+1, j+1)
\end{array}\right) / 4 \\
& \text { else } \\
& \text { end } \\
& \text { else } \\
& \text { end } \\
& \varepsilon=0.000001 \text {; } \\
& \mathrm{dt}=0.1 ; \\
& {\left[\mathrm{I}_{\mathrm{x}}, \mathrm{I}_{\mathrm{y}}\right]=\text { gradient(gradient(output)); }} \\
& \mathrm{c}=1 . /\left(\operatorname{sqrt}\left(\mathrm{I}_{\mathrm{x} .} \wedge 2+\mathrm{I}_{\mathrm{y}} \wedge 2\right)+\varepsilon\right) ; \\
& {[\operatorname{div} 1, \operatorname{div} 2]=\operatorname{gradient}\left(\operatorname{gradient}\left(\operatorname{c} . * \mathrm{I}_{\mathrm{x}}\right)\right) \text {; }}
\end{aligned}
$$

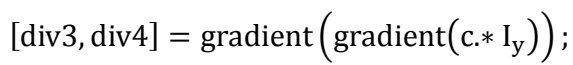

$$
\begin{aligned}
& \operatorname{div}=\operatorname{div} 1+\operatorname{div} 4 ; \\
& \text { output }=\text { output (dt.* div); } \\
& \text { op = output; } \\
& \text { where gradient }=\left(\sqrt{M_{x}^{2}+M_{y}^{2}}\left(q_{3}\right)\right) \text {; }
\end{aligned}
$$

\section{Step10: Get final image}

\subsection{Results and Discussions Performance Evaluation}

To compare the proposed method with [S.Alireza Golestaneh et al., 2014] on the basis on the following parameters:

\section{Peak Signal to Noise Ratio(PSNR)}

It is used to evaluate the quality of the image. PSNR is the ratio between the maximum possible power of a signal and the power of degrading noise that affects the constancy of its representation.

PSNR $=10 \log _{10} \frac{\left(2^{\mathrm{b}}-1\right)^{2}}{\mathrm{MSE}} d B$

Where $b=$ no of bits per pixel (bpp)

MSE $=$ Mean square error

PSNR needs to be maximized for better results. Table1 has clearly shown the PSNR maximum in the case of proposed method. So the proposed method has better results than the available methods.

Table 1 is Showing the Comparative Analysis of the Peak Signal to Noise Ratio (PSNR)

\begin{tabular}{|l|l|l|}
\hline $\begin{array}{l}\text { Test } \\
\text { Images }\end{array}$ & $\begin{array}{l}\text { Existing method [S.Alireza } \\
\text { Golestaneh et al., 2014] }\end{array}$ & $\begin{array}{l}\text { Proposed } \\
\text { method }\end{array}$ \\
\hline 1. & 29.3929 & 30.5030 \\
\hline 2. & 25.4528 & 27.9001 \\
\hline 3. & 31.5594 & 32.6183 \\
\hline 4. & 25.1133 & 27.2824 \\
\hline 5. & 28.1790 & 28.8948 \\
\hline 6. & 27.0147 & 27.8631 \\
\hline 7. & 30.0930 & 31.0315 \\
\hline 8. & 29.9031 & 30.1224 \\
\hline
\end{tabular}

\section{Mean Square Error (MSE)}

It is cumulative square error between compressed image and referenced image.

$\mathrm{MSE}=\frac{1}{\mathrm{MN}} \sum_{\mathrm{m}=1}^{\mathrm{M}} \sum_{\mathrm{n}=1}^{\mathrm{N}}[\mathrm{I}(\mathrm{m}, \mathrm{n})-\mathrm{F}(\mathrm{m}, \mathrm{n})]^{2}$

Where $I(m, n)$ is the pixel value of referenced image

$\mathrm{F}(\mathrm{m}, \mathrm{n})$ is pixel value of compressed image

Table 2 Depicts the Analysis of the Mean Square Error. As Mean Square Error needs to be reduced. So the Proposed method shows the better results than the available method

\begin{tabular}{|l|l|l|}
\hline $\begin{array}{l}\text { Test } \\
\text { Images }\end{array}$ & $\begin{array}{l}\text { Existing method [S.Alireza } \\
\text { Golestaneh et al., 2014] }\end{array}$ & $\begin{array}{l}\text { Proposed } \\
\text { method }\end{array}$ \\
\hline 1. & 74.7815 & 57.9138 \\
\hline 2. & 185.2694 & 105.4565 \\
\hline 3. & 45.4087 & 35.5836 \\
\hline 4. & 200.3338 & 121.5732 \\
\hline 5. & 98.8964 & 83.8696 \\
\hline 6. & 129.3049 & 106.3572 \\
\hline
\end{tabular}




\begin{tabular}{|l|l|l|}
\hline 7. & 63.6480 & 51.2783 \\
\hline 8. & 66.4928 & 63.2175 \\
\hline
\end{tabular}

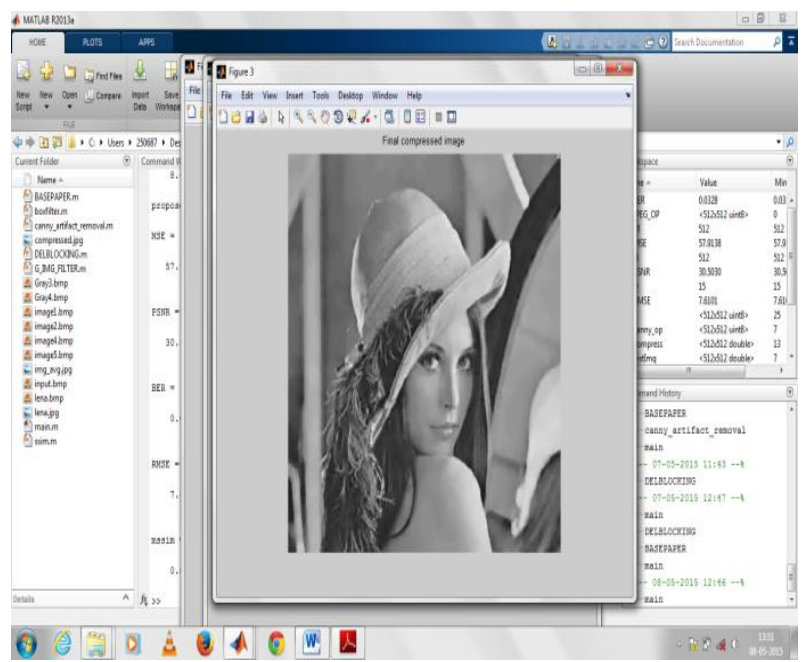

(a)

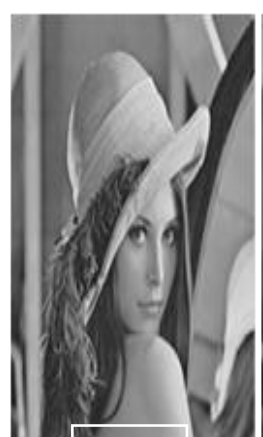

(b)

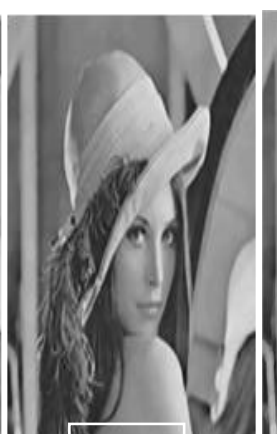

(c)

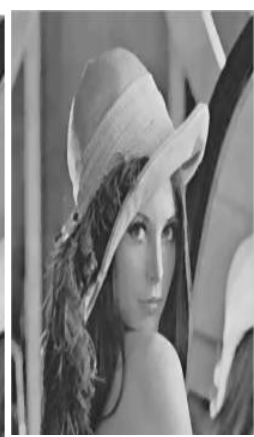

(d)
Fig. 6 (a) Screenshot of proposed algorithm (b) Original image (c) JPEG compression and Edge regeneration for artefacts removal (d) Final image by proposed method

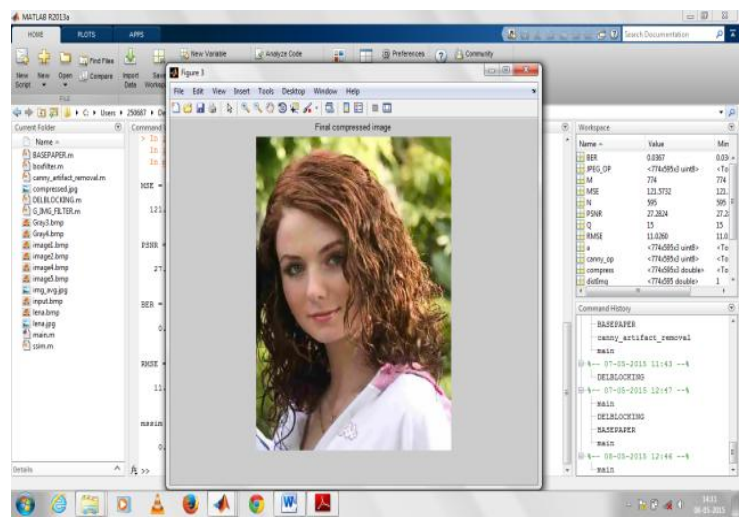

(a)

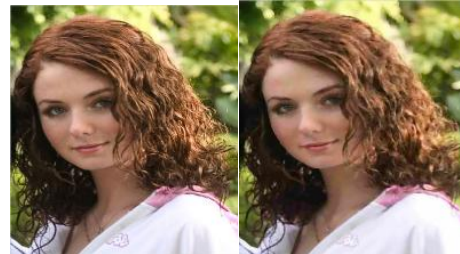

(b) (c)

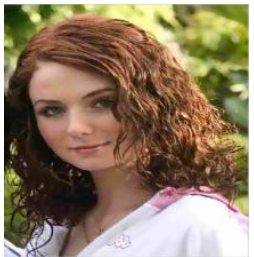

(d)
Fig. 7(a) Screenshot of proposed algorithm (b) Input image (c) JPEG Compression with edge regeneration (d) Final image by proposed method.

\section{CONCLUSION}

This paper presents a new method for artefacts removal from compressed image. Artefacts round the image degrade the visual quality of image. Restoration method as a post processing based on canny edge detections used to attain the nice visual quality outcomes of the image. Compression of image encounters several types of artefacts in resultant image. This paper successfully improves the visual outcomes of the compressed image. This achieves better results than available method.

\section{REFERENCES}

[1] G. K. Wallace 1991 The JPEG still picture compression standard, communication ACM.

[2] Giridhat mandhyam et al.1995 DCT based scheme for lossless image compression.

[3] Sonal, Various image compression techniques, IJCS.

[4] Subramanya A. 2001 Image Compression Technique

[5] A. Nosratinia, 2003 Post-processing of JPEG images to remove compression artifacts IEEE.

[6] J.S. Walker, T.Q. Nguyen, Y. Chen 2003 A low-power, low memory system for wavelet-based image compression IEEE.

[7] A. W. C. Liew and H. Yan 2004 Blocking artifacts suppression in block-coded images using over-complete wavelet representation IEEE.

[8] Ying Luo and Rabab K. Ward 2004 Removing the blocking artifacts of block-based DCT compressed images IEEE.

[9] Arun Kumar PS 2007 Implementation of Image Compression Algorithm using Verilog with Area, Power and Timing Constraints.

[10] J. Kim et al.2007 Reduction of blocking artifacts for HDTV using offset-and-shift technique IEEE.

[11] R.C. Gonzalez and R.E. Woods 2007 Digital Image processing.

[12] S. Singh et al.2007 Reduction of blocking artifacts in JPEG compressed images, Digital signal processing.

[13] Guangtao Zhai et al.2008 Image de-ringing using quadtree based block shift filtering, IEEE.

[14] Guangtao Zhai et al.2008 Efficient image deblocking based on postfiltering in shifted windows IEEE.

[15] J. Singh et al. 2011, A signal adaptive filter for blocking effect reduction of JPEG compressed images, AEU Int. J. Electronics communication. 
[16] Ke Du Haiyun Han and Gang Wang 2011 A new algorithm for removing compression artifacts of waveletbased image IEEE.

[17] Prabhakar.Telagarapu et al.2011 Image compression using DCT and wavelet transformations.

[18] C. Jung n et al. 2012 Image de-blocking via sparse representation, Signal processing: Image Communication.

[19] E. Kannan et al.2012 Lossless Image Compression Algorithm For Transmitting Over Low Bandwidth Line.

[20] Mahmud Hasan 2012 Spatial Domain Lossless Image Compression Technique by Reducing Overhead Bits and
Run Length Coding (IJCSIT) International Journal of Computer Science and Information Technologies.

[21] Gaganpreet et al.2013 Comparative Analysis of Various Digital Image Compression Techniques Using Wavelets

[22] S. Alireza and Damon M. Chandler 2014 An algorithm for JPEG artifacts reduction via local edge regeneration.

[23] Reza Pourreza-Shahri et al. 2014 A gradient based optimization approach for reduction of blocking artifactss in JPEG images, IEEE. 